\title{
Stroke Severity Is the Major Player in Post-Stroke Urinary Tract Infection in Patients with First Ever Ischemic Stroke
}

\author{
Hassan Mohamed Elnady', Hemaid Mostafa Azab', Mohamed Abdelmonem Said', \\ Alaa-Eldin Sedky Bekheet ${ }^{1}$, Ashraf Khodeary ${ }^{2}$, Ahmed Ata Ali ${ }^{1}$ \\ ${ }^{1}$ Department of Neurology, Sohag University, Sohag, Egypt \\ ${ }^{2}$ Department of Clinical Pathology, Sohag University, Sohag, Egypt \\ Email: hass_elnady@yahoo.com
}

How to cite this paper: Elnady, H.M., Azab, H.M., Said, M.A., Bekheet, A.-E.S., Khodeary, A. and Ali, A.A. (2018) Stroke Severity Is the Major Player in Post-Stroke Urinary Tract Infection in Patients with First Ever Ischemic Stroke. Neuroscience \& Medicine, 9, 94-104.

https://doi.org/10.4236/nm.2018.92010

Received: March 6, 2018

Accepted: June 25, 2018

Published: June 29, 2018

Copyright $\odot 2018$ by authors and Scientific Research Publishing Inc. This work is licensed under the Creative Commons Attribution International License (CC BY 4.0).

http://creativecommons.org/licenses/by/4.0/

(c) (i) Open Access

\begin{abstract}
Background: Urinary tract infections UTIs occur repeatedly after stroke and are related to bad outcomes with increased rates of deterioration in neurological state during hospitalization, death or long term disability as well as increased length of hospitalization. Factors found to predict UTI include stroke severity, depressed consciousness level, increased post-void residual urine volume, and diabetes mellitus. Stroke severity appears to be the most important predictor of infection risk. We aimed to determine the risk factors associated with UTI after acute stroke, and its association with outcome. Subjects and Methods: This is prospective cohort study. We analysed clinical data of 100 patients with first ever ischemic stroke. We assessed risk factors for UTI, as well as clinical outcome. Results: Urinary tract infection was found in $72 \%$ of our subjects. On univariate analysis, patients with UTI were more likely to have had a more severe stroke, more likely to be catheterized and more likely to have a higher serum creatinine level. The multivariate analysis revealed that greater stroke severity was independently associated with increased risk of developing UTI. Greater stroke severity measured by CSS was independently associated with unfavorable outcome on discharge. Conclusion: UTI is common after acute stroke. It is associated with more severe stroke.
\end{abstract}

\section{Keywords}

Acute Stroke, Urinary Tract Infection, Stroke Severity

\section{Introduction}

Stroke remains a tremendous public heath burden with approximately 795,000 
people affected yearly. Stroke is the leading cause of major long-term disability in adults and the third leading cause of death in developed countries [1]. Medical complications are frequent in the post stroke period and can prolong the duration of hospital admission, worsen stroke outcomes, and increase the economic burden of care [2]. Infections, including pneumonia and urinary tract infection (UTI), are among the most common medical complications in the post stroke period [3]. Urinary tract infections UTIs occur repeatedly after stroke and are related to bad outcomes with increased rates of deterioration in neurological state during hospitalization, death or long term disability as well as increased length of hospitalization [4].

Infections can result in electrolyte disturbances, hypoxia, and elevated body temperature, which have, theoretically, a negative effect on vitality of neurons within the ischemic penumbra [5]. Fever may increase the cerebral metabolic demands, change the blood-brain barrier permeability, and promote acidosis and release of excitatory amino acids [6].

The revealed risk of hospital acquired UTI varies significantly in the available studies with risks ranging from $3.7 \%$ to $65.8 \%$. Differences in study populations, methodology and definition of infection may be the cause for these considerable variations in post stroke infection rates [7]. The majority of in-hospital UTIs are associated with catheterization but whether catheterized or not, patients with stroke have a higher rate of UTI when compared with the general medical and surgical subjects [8]. Factors found to predict UTI include stroke severity, depressed consciousness level, increased post-void residual urine volume, and diabetes mellitus [7] [9].

A more severe stroke appears to be the most powerful predictor of infection risk. Basic research studies, suggest that ischemic brain insult may lead to a "systemic immunodepression" that predisposes to infection. In addition, stroke severity was a strong predictor for the degree of lymphopenia occurring during infection [9] [10] [11].

Some reports suggest that antibiotics therapy in the early stages of stroke (even in non infected patients) minimizes cerebral ischemia and ameliorates the outcome [12] [13].

We aimed to determine the incidence of UTI after acute stroke, the risk factors associated with this complication, and its association with outcome.

\section{Patients and Methods}

This prospective cohort study included 100 patients who were admitted to the department of Neurology, Sohag university hospital in the period between october 2012 and December 2013. The inclusion criterion was first ever acute ischemic stroke within 7 days of onset.

Excluded from this study were patients with recurrent stroke, hemorrhagic stroke, subarachnoid hemorrhage, UTI manifestations or treatment immediately before stroke insult. Patients or their relatives consented to this study, which was 
approved by the scientific and ethical committee at Sohag faculty of medicine.

Stroke was defined according to the definition of the World Health Organization as the rapid onset of a new persistent neurological deficit attributable to an obstruction in cerebral blood flow with no apparent non-vascular cause [14].

The participants of the current study were subjected to detailed medical and neurological evaluation. Admission stroke severity was assessed by Canadian Stroke Scale (CSS).

The etiology of ischemic stroke (IS) was determined according to the criteria developed for the Trial of Org 10172 in Acute Stroke Treatment. The five categories of IS were isolated according to etiology, that is, 1) large artery atherosclerosis, 2) cardioembolism, 3) small artery occlusion, 4) other determined etiology, and 5) unknown or multiple etiologies [15].

laboratory investigations including: Electrolyte assessment $(\mathrm{Na}+\mathrm{K}+\mathrm{Ca}++)$, random blood sugar, liver functions, renal functions, lipid profile, complete blood count (CBC) and erythrocyte sedimentation rate (ESR), urine analysis, and Urine culture on admission and 2 and 7 days later, and CT or MRI brain.

Outcome on discharge using the Modified Rankin score (MRS) was chosen as the outcome measure for the subsequent statistical analysis. Patients with Modified Rankin Score of $\leq 2$ were considered to have favorable outcome, while those with Modified Rankin Score of $>2$ were considered to have Unfavorable outcome.

\subsection{Laboratory Investigations}

\section{1) Sampling:}

We obtained both blood and urine samples from all patients. Firstly we took 7 $\mathrm{ml}$ venous blood by venipuncture and divided in 3 tubes, the first was EDTA vacutainer for CBC, the second was ESR tube for ESR, and the third was plane vacutainer for chemical investigations. Secondly we obtained midstream urine sample in sterile cup for complete urine analysis and microbiological studies but at first we were sure that no antibiotic therapy in the last 48 hours.

$\mathrm{CBC}$ was done by celtac automated hematology analyzer after calibration. ESR was done by westergreen tube method.

Chemical examinations included random blood sugar, urea, creatinine, liver function tests (bilirubin, AST, ALT, alkaline phospahtase, albumin, total protein), electrolytes ( $\mathrm{Na}, \mathrm{K}, \mathrm{Calcium})$ and lipid profile. These chemical investigations were done by Bechman Synchron CX9 automated system.

\section{2) Microbiological studies:}

We did complete urine analysis then we inoculated the sample in nutrient agar, blood agar and Mac conkey agar to obtain separate pure colonies to be identified by VITEK automated identification microbiology system and according to the manufacture manual.

The VITEK System is an automated microbiology system utilizing growthbased technology. It uses colorimetric reagent cards that are incubated and in- 
terpreted automatically.

\section{3) The viable bacterial count:}

The dip-slides with Mac Conkey agar on one side and nutrient agar on the other were used according to the manufacturer's instructions. The viable count was assessed by comparing the density of growth on each medium with the manufacturer's standard charts. The diagnosis of UTI is established when the count is $\geq 10^{5}$ bacteria per milliliter of urine.

\subsection{Statistical Analysis}

Analysis of data was carried out using the "Statistical Program for Social Sciences" (SPSS) for windows version 16.0.Categorical variables were expressed as frequency and percentage while continuous variables were expressed as mean \pm standard deviation. Pearson Chi-square and independent student's ( $t$ ) test were used in univariate analysis. The threshold level for statistical significance was established at $P<0.05$. Logistic regression analysis was employed to find the best predictive model. Variables with a $p$ value $<0.05$ in the univariate analyses were included as covariates.

\section{Results}

A total of 100 patients with first ever acute ischemic stroke were included in the study comprising $38(38 \%)$ males and 62 (62\%) females. The mean age "mean \pm SD" in our subjects was $62.27 \pm 13.1$. The baseline characteristics of the subjects are presented in (Table 1 ).

The subjects were classified into two groups according to the presence or absence of UTI. The percentage of patients with UTI was $72 \%$ and of those without UTI was $28 \%$. Figure 1 show the percentage of different infectious agents among stroke patients with UTI.

Gram positive cocci (mainly Staphylococci and Enterococci) were the most common pathogenic organisms and accounted for 35\%. Mixed infection (Gram positive cocci and Gram negative bacilli) also accounted for $21 \%$ of the UTIs. The percentage of Candida and Gram negative bacilli infections was $9 \%$ and $7 \%$ respectively. Esc. coli, Proteus species and Pseudomonas aerioginosa were the commonest gram negative bacilli.

On univariate analysis, patients with UTI were more likely to have had a more severe stroke as shown by their CSS on admission when compared with those who did not develop a UTI $(p=0.010)$, and they were also more likely to have had a urinary catheter $(p=0.012)$ (Table 2$)$. There were also statistically significant difference in the serum creatinine level, which was higher in those diagnosed with UTI when compared with those who had no UTI $(p=0.041)$. The univariate analysis revealed no statistically significant differences between the two groups when comparing their age, gender, history of diabetes, history of hypertension, maximum temperature on admission or the type of IS according to the TOAST criteria (Table 2). 


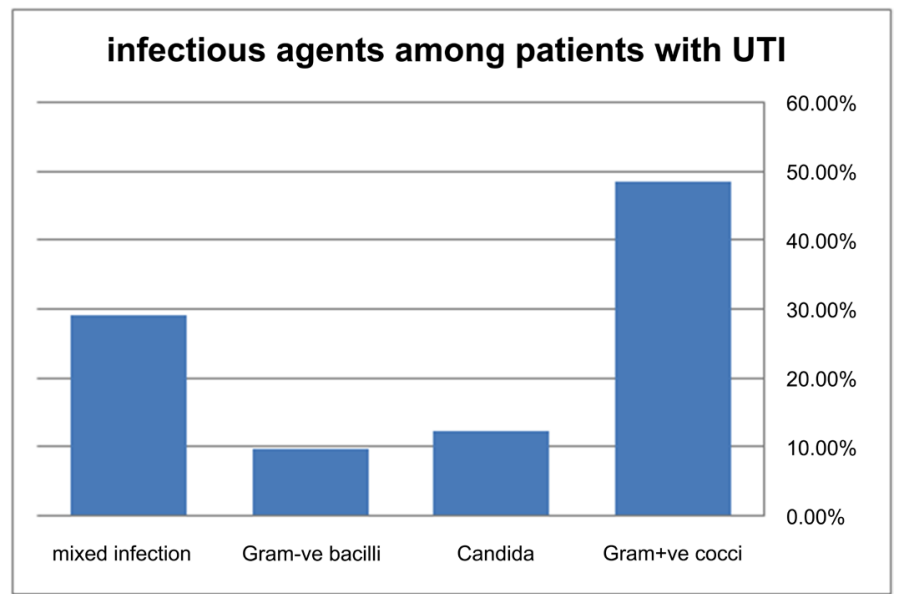

Figure 1. Percentage of infectious agents among stroke patients with UTI.

Table 1. Baseline characteristics of patients.

\begin{tabular}{lc}
\hline Age, mean, SD (years) & $62.2 \pm 13.001$ \\
\hline Gender & $38(38)$ \\
Male, $\mathrm{n}(\%)$ & $62(62)$ \\
Female, $\mathrm{n}(\%)$ & $42(42)$ \\
Hypertension, $\mathrm{n}(\%)$ & $22(22)$ \\
Diabetes, $\mathrm{n}(\%)$ & $2(2)$ \\
Previous TIA, $\mathrm{n}(\%)$ & $17(17)$ \\
Cardiac Disease, n (\%) & \\
Etiology of IS (TOAST) & $47(47)$ \\
Small vessel disease, $\mathrm{n}(\%)$ & $32(32)$ \\
Large artery disease, $\mathrm{n}(\%)$ & $13(13)$ \\
Cardio embolic, $\mathrm{n}(\%)$ & $8(8)$ \\
Undetermined cause, $\mathrm{n}(\%)$ & $6.16 \pm 3.4$ \\
CSS, mean, SD & \\
Outcome & $43(43)$ \\
MRS $\leq 2, \mathrm{n}(\%)$ & $56(56)$ \\
MRS $>2, \mathrm{n}(\%)$ &
\end{tabular}

UTI: Urinary tract infection; No: the number of patients; SD: standard deviation; TIA: transient ischemic attack; TOAST: Trial of Org 10172 in Acute Stroke Treatment; CSS: Canadian Stroke Scale; MRS: modified Rankin Scale.

Table 2. Risk factors, clinical characteristics, and laboratory values in patients with and without UTI.

\begin{tabular}{lccc}
\hline \multicolumn{1}{c}{ Characteristics } & With UTI No. (72) & Without UTI No. (28) & $P$ value \\
\hline $\begin{array}{l}\text { Age, mean, SD (years) } \\
\text { Gender }\end{array}$ & $58.29 \pm 11.19$ & $63.81 \pm 13.39$ & 0.056 \\
$\quad$ Male, n (\%) & $29(40.3 \%)$ & $9(32.1 \%)$ & 0.457 \\
$\quad$ Female, n (\%) & $43(59.7 \%)$ & $19(67.9 \%)$ & \\
Hypertension, n (\%) & $31(43.1 \%)$ & $11(39.9 \%)$ & 0.735 \\
Diabetes, n (\%) & $15(20.8 \%)$ & $7(25.0 \%)$ & 0.655 \\
Previous TIA, n (\%) & $2(2.8 \%)$ & $0(0.0 \%)$ & 0.378 \\
\hline
\end{tabular}




\section{Continued}

\begin{tabular}{lccc}
\hline Cardiac Disease, n (\%) & $14(19.4 \%)$ & $3(10.7 \%)$ & 0.301 \\
Etiology of IS (TOAST) & & & \\
$\quad$ Small vessel disease, n (\%) & $17(36.1 \%)$ & $30(63.8 \%)$ & 0.174 \\
$\quad$ Large artery disease, n (\%) & $8(25 \%)$ & $24(75 \%)$ & \\
$\quad$ Cardio embolic, n (\%) & $3(23.07 \%)$ & $10(76.9 \%)$ & \\
$\quad$ Undetermined cause, n (\%) & $0(0 \%)$ & $8(100 \%)$ & 0.38 \\
Body temperature, mean, SD & $37.57 \pm 0.58$ & $37.69 \pm 0.75$ & 0.010 \\
CSS, mean, SD & $5.6 \pm 3.3$ & $7.5 \pm 3.3$ & 0.012 \\
Catheterization & $64(77.1 \%)$ & $19(22.9 \%)$ & 0.457 \\
Blood sugar, mean, SD & $155.4 \pm 65.83$ & $144.96 \pm 54.1$ & 0.041 \\
Serum creatinine, mean, SD & $1.33 \pm 0.73$ & $1.03 \pm 0.28$ & 0.617 \\
RBCs, mean, SD & $4.56 \pm 0.64$ & $4.64 \pm 0.70$ & 0.671 \\
HB, mean, SD & $13.42 \pm 4.16$ & $13.33 \pm 3.61$ & 0.268 \\
W BCs, mean, SD & $10.41 \pm 3.72$ & $4.47 \pm 3.87$ & 0.703 \\
Platelets, mean, SD & $222.76 \pm 44.62$ & $218.75 \pm 53.03$ & 0.816 \\
ESR, mean, SD & $29.69 \pm 17.15$ & $28.79 \pm 18.21$ & 0.369 \\
Cholesterol, mean, SD & $190.58 \pm 55.71$ & $179.50 \pm 53.67$ & 0.434 \\
Uric acid, mean, SD & $7.37 \pm 1.98$ & $7.27 \pm 2.07$ & 0.743 \\
Serum albumin, mean, SD & $3.32 \pm 0.39$ & $3.34 \pm 0.33$ & \\
\hline
\end{tabular}

UTI: Urinary tract infection; No: the number of patients; SD: standard deviation; TIA: transient ischemic attack; TOAST: Trial of Org 10172 in Acute Stroke Treatment; CSS: Canadian Stroke Scale; RBCs: Red blood cells; HB: Hemoglobin; W BCs: White blood cells; ESR: Erythrocyte Sedimentation Rate.

The data were further analyzed by binary logistic regression (Table 3 ). The dependent variable was the presence or absence of UTI, and the independent variables were stroke severity measured by CSS, the presence or absence of a urinary catheter, and the serum creatinine level. This analysis revealed that, greater stroke severity measured by CSS to be independently associated with increased risk of developing UTI $(p=0.013)$.

To determine the impact of urinary tract infection on the outcome of the ischemic stroke, the subjects were further divided into two groups; those with favorable outcome and those with unfavorable outcome (Table 4). Patients in the favorable outcome group are those with $\mathrm{MRS} \leq 2$, while patients with the unfavorable outcome are those with MRS $>2$. On univariate analysis, patients with unfavorable outcome were more likely to develop post stroke UTI ( $p=$ 0.002 ), and have had a more severe stroke as shown by their CSS on admission $(p<0.00001)$ when compared with those favorable outcome. Patients with favorable outcome were less likely to be catheterized $(p<0.00001)$. Logistic regression analysis was utilized to determine the best predictive model of poor outcome. This analysis revealed that greater stroke severity measured by CSS to be independently associated with unfavorable outcome on discharge $(p<$ 0.00001) (Table 5). 
Table 3. Logistic regression model of post-stroke urinary tract infection in patients with first ever ischemic stroke.

\begin{tabular}{lcccc}
\hline & Exp (B) & \multicolumn{2}{c}{$95 \%$ C. I. for EXP (B) } & $P$-value \\
\hline & & Lower & upper & \\
CSS on admission & 0.838 & 0.729 & 0.963 & 0.013 \\
\hline
\end{tabular}

CI: confidence interval; CSS: Canadian stroke scale score.

Table 4. Risk factors, clinical characteristics, and laboratory values in patients with favorable and unfavorable outcome.

\begin{tabular}{|c|c|c|c|}
\hline Characteristics & $\begin{array}{l}\text { Favorable outcome } \\
\text { MRS } \leq 2 \text { No. }(72)\end{array}$ & $\begin{array}{l}\text { Unfavorable outcome } \\
\text { MRS }>2 \text { No. }(28)\end{array}$ & $P$ value \\
\hline Age, mean, SD (years) & $60.1 \pm 13.5$ & $63.9 \pm 12.4$ & 0.147 \\
\hline \multicolumn{4}{|l|}{ Gender } \\
\hline Male, n (\%) & $20(52.6 \%)$ & $18(47.3 \%)$ & 0.128 \\
\hline Female, n (\%) & $23(37.1 \%)$ & $39(62.9 \%)$ & \\
\hline Hypertension, n (\%) & $17(40.4 \%)$ & $25(59.5 \%)$ & 0.668 \\
\hline Diabetes, n (\%) & $11(50 \%)$ & $11(50 \%)$ & 0.45 \\
\hline \multicolumn{4}{|l|}{ Etiology of IS (TOAST) } \\
\hline Small vessel disease, $\mathrm{n}(\%)$ & $22(46.8 \%)$ & $25(53.2 \%)$ & 0.29 \\
\hline Large artery disease, $\mathrm{n}(\%)$ & $13(40.6 \%)$ & $19(59.3 \%)$ & \\
\hline Cardio embolic, n (\%) & $3(23.07 \%)$ & $10(76.9 \%)$ & \\
\hline Undetermined cause, $\mathrm{n}(\%)$ & $5(62.5 \%)$ & $3(37.5 \%)$ & \\
\hline Body temperature, mean, SD & $37.63 \pm 0.6$ & $37.58 \pm 0.59$ & 0.655 \\
\hline UTI, n (\%) & $24(33.3 \%)$ & $48(77.4)$ & 0.002 \\
\hline Catheterization & $20(27.1 \%)$ & $54(72.9 \%)$ & $<0.00001$ \\
\hline CSS, mean, SD & $8.5 \pm 2.7$ & $4.3 \pm 2.7$ & $<0.00001$ \\
\hline Blood sugar, mean, SD & $157.6 \pm 71.1$ & $148.5 \pm 55.7$ & 0.474 \\
\hline Serum creatinine, mean, SD & $1.2 \pm 0.5$ & $1.2 \pm 0.7$ & 0.87 \\
\hline RBCs, mean, SD & $4.6 \pm 0.6$ & $4.5 \pm 0.6$ & 0.45 \\
\hline W BCs, mean, SD & $10.2 \pm 3.9$ & $10.1 \pm 3.6$ & 0.8 \\
\hline Platelets, mean, SD & $216.1 \pm 45.4$ & $225.8 \pm 47.8$ & 0.3 \\
\hline ESR, mean, SD & $27.2 \pm 14.9$ & $31.1 \pm 18.9$ & 0.272 \\
\hline Cholesterol, mean, SD & $189.3 \pm 54.8$ & $186.1 \pm 55.7$ & 0.77 \\
\hline
\end{tabular}

MRS: modified Rankin Scale; No: the number of patients; SD: standard deviation; TOAST: Trial of Org 10172 in Acute Stroke Treatment; UTI: Urinary tract infection; CSS: Canadian Stroke Scale; RBCs: Red blood cells; HB: Hemoglobin; WBCs: White blood cells; ESR: Erythrocyte Sedimentation Rate.

Table 5. Logistic regression model of outcome on discharge in patients with favorable and unfavorable outcome.

\begin{tabular}{ccccc}
\hline & Exp (B) & \multicolumn{2}{c}{$95 \%$ C. I. for EXP (B) } & $P$-value \\
\hline \multirow{3}{*}{ CSS on admission } & 0.627 & Lower & upper & \\
\hline
\end{tabular}

CI: confidence interval; CSS: Canadian stroke scale score. 


\section{Discussion}

In this study, UTI occurred in $72 \%$ of patients following an acute stroke. Previous studies in stroke patients have cited an extensive variety of rates for UTI, from as low as $3 \%$ up to as high as $44 \%$ [16]. Indredavik and colleagues reported that hospital incidence of UTIs are very common among stroke patients of $16 \%$ in the first week and $27.9 \%$ at 3 months [9]. Davenport and colleagues found an in-hospital UTI incidence of $16 \%$ in 613 consecutive acute stroke admissions [17]. Also with a multicenter study of 311 consecutive acute stroke admissions, the in-hospital incidence of UTI was $24 \%$ [18]. In another previous study of 1455 patients of acute ischemic stroke patients recruited to a randomized controlled trial, the authors found an incidence of $17.2 \%$ of UTI [10]. Stott and colleagues denoted that the wide variations of in-hospital UTI rates in stroke patients are likely to be a result of differences in selection of patients and in case $\operatorname{mix}[7]$.

We have found, in both univariate and multivariate analysis, that stroke severity was a strong predictor for the development of post stroke UTI. This finding is online with the previous study of Indredavik and colleagues who reported that, severity of stroke as assessed by reduced consciousness on the Scandinavian Stroke Scale at admission was significantly associated with an increased risk for the development of UTI [9]. Our data also corroborate the previous findings that T-lymphopenia is detectable within hours after stroke onset [19] [20] and that its severity correlates with the risk of infection [21].

Moreover, Tanzi and colleagues found that stroke severity is the most important predictor of infection risk following stroke [22]. The authors also, reported that patients with more severe strokes may be at higher risk of infection due to exposure to more indwelling catheters, but experimental data also suggest that strokes induce systemic changes in the immune response that predispose to infection [22]. A previous experimental data, suggest that ischemic brain injury may lead to a "systemic immunodepression" that predisposes to infection [23]. In animal studies, this immunodepression is characterized by impaired lymphocyte function, which appears to be sympathetically mediated [23].

The isolation rate of urinary pathogens is inconsistent with reports of other previously published studies [24] [25] [26]. The cause of that difference is the hospital acquired nature of the infection in our subjects and presence of urinary catheter in a higher percentage of them.

The high percentage of Candida infection in our study is likely related to immunodepression which is proved from previous studies [27].

We have found that urinary catheterization, in univariate analysis, to be associated with post-stroke UTI. This finding is consistent with previous studies [7] [8]. On the other hand we did not find any association between urinary catheterization and post-stroke UTI in the multivariate analysis. This observation is in disagreement with that of Stott and colleagues, who reported that catheterisation is independently associated with increased risk of developing UTI in both univa- 
riate and multivariate analysis [7]. The link between urinary catheter and UTI has been discussed in several previous studies. Kunin and colleagues, reported that avoidance of unnecessary catheterization is likely to be the single most effective strategy in preventing UTI [28]. Other authors, advised that if a urinary catheter is used it may be possible to reduce the risk of associated infection by early removal, attention to detail in catheter-care or by use of modified catheters coated with antimicrobials [7]. A programmed reminder to nurses by physicians to remove unnecessary catheters has been shown to reduce duration of catheterization and associated infections [29].

Our study-in the univariate analysis-identified a statistically significant difference in the serum creatinine level, which was higher in patients with UTI. Our explanation of this finding is that, acute kidney injury is frequently encountered in patients with acute ischemic stroke [30] such effect may increase in stroke patients with other comorbidity. However, we did not find this association in the multivariate analysis.

Demographic (age and gender) and vascular (hypertension, diabetes, and cardiac disease) risk factors were not related to the occurrence of post stroke UTI in our analysis. Regarding these findings, there are contradictory results from the previous studies. In two previous studies age was reported as a risk factor of poststroke UTI [7] [10]. However, Donkor and colleagues did not find age as a risk factor of poststroke UTI [31]. Sex was not associated with post-stroke UTI in the study of Stott and colleagues [7] while female preponderance was found in the study of Aslanyan and colleagues [10]. A previous authors claimed that diabetes mellitus has an independent association with UTI [32], while others did not [7].

Our study was in agreement with previous studies [7] [22] which reported that post stroke UTI was associated with unfavorable outcome. However we did find this effect only in the univariate analysis. In the multivariate analysis, stroke severity was the only independent risk factor for worse outcome.

Our study does have a number of limitations. Firstly, the small number of patients which needs further investigations on a larger number of patients. Secondly, we did not employ strict pre-defined criteria for UTI diagnosis. Thirdly, the short duration of follow up limits identification of the accurate effect of poststroke UTI on stroke outcome.

\section{References}

[1] Lee, M., et al. (2007) First-Ever Ischemic Stroke in Very Old Asians: Clinical Features, Stroke Subtypes, Risk Factors and Outcome. European Neurology, 58, 44-48. https://doi.org/10.1159/000102166

[2] Weimar, C., et al. (2002) Complications Following Acute Ischemic Stroke. European Neurology, 48, 133-140. https://doi.org/10.1159/000065512

[3] Dromerick, A.W. and Edwards, D.F. (2003) Relation of Postvoid Residual to Urinary Tract Infection during Stroke Rehabilitation. Archives of Physical Medicine and Rehabilitation, 84, 1369-1372. https://doi.org/10.1016/S0003-9993(03)00201-6 
[4] Rocco, A., et al. (2007) Monitoring after the Acute Stage of Stroke: A Prospective Study. Stroke, 38, 1225-1228. https://doi.org/10.1161/01.STR.0000259659.91505.40

[5] Chamorro, A., Urra, X. and Planas, A.M. (2007) Infection after Acute Ischemic Stroke: A Manifestation of Brain-Induced Immunodepression. Stroke, 38, 1097-1103. https://doi.org/10.1161/01.STR.0000258346.68966.9d

[6] Greer, D.M., et al. (2008) Impact of Fever on Outcome in Patients with Stroke and Neurologic Injury: A Comprehensive Meta-Analysis. Stroke, 39, 3029-3035. https://doi.org/10.1161/STROKEAHA.108.521583

[7] Stott, D.J., et al. (2009) Urinary Tract Infection after Stroke. QJM, 102, 243-249. https://doi.org/10.1093/qjmed/hcp012

[8] Ersoz, M., et al. (2007) Urinary Tract Infection and Bacteriurua in Stroke Patients: Frequencies, Pathogen Microorganisms, and Risk Factors. American Journal of Physical Medicine \& Rehabilitation, 86, 734-741. https://doi.org/10.1097/PHM.0b013e31813e5f96

[9] Indredavik, B., et al. (2008) Medical Complications in a Comprehensive Stroke Unit and an Early Supported Discharge Service. Stroke, 39, 414-420.

https://doi.org/10.1161/STROKEAHA.107.489294

[10] Aslanyan, S., et al. (2004) Pneumonia and Urinary Tract Infection after Acute Ischaemic Stroke: A Tertiary Analysis of the GAIN International Trial. European Journal of Neurology, 11, 49-53. https://doi.org/10.1046/j.1468-1331.2003.00749.x

[11] Walter, U., et al. (2007) Predictors of Pneumonia in Acute Stroke Patients Admitted to a Neurological Intensive Care Unit. Journal of Neurology, 254, 1323-1329. https://doi.org/10.1007/s00415-007-0520-0

[12] Lampl, Y., et al. (2007) Minocycline Treatment in Acute Stroke: An Open-Label, Evaluator-Blinded Study. Neurology, 69, 1404-1410. https://doi.org/10.1212/01.wnl.0000277487.04281.db

[13] Schwarz, S., et al. (2008) Effects of Prophylactic Antibiotic Therapy with Mezlocillin plus Sulbactam on the Incidence and Height of Fever after Severe Acute Ischemic Stroke: The Mannheim Infection in Stroke Study (MISS). Stroke, 39, 1220-1227. https://doi.org/10.1161/STROKEAHA.107.499533

[14] Hatano, S. (1976) Experience from a Multicentre Stroke Register: A Preliminary Report. Bull World Health Organ, 54, 541-553.

[15] Adams Jr., H.P., et al. (1993) Classification of Subtype of Acute Ischemic Stroke. Definitions for Use in a Multicenter Clinical Trial. TOAST. Trial of Org 10172 in Acute Stroke Treatment. Stroke, 24, 35-41. https://doi.org/10.1161/01.STR.24.1.35

[16] Bogason, E., et al. (2017) Urinary Tract Infections in Hospitalized Ischemic Stroke Patients: Source and Impact on Outcome. Cureus, 9, e1014.

[17] Davenport, R.J., et al. (1996) Complications after Acute Stroke. Stroke, 27, 415-420. https://doi.org/10.1161/01.STR.27.3.415

[18] Langhorne, P., et al. (2000) Medical Complications after Stroke: A Multicenter Study. Stroke, 31, 1223-1229. https://doi.org/10.1161/01.STR.31.6.1223

[19] Chamorro, A., et al. (2006) Interleukin 10, Monocytes and Increased Risk of Early Infection in Ischaemic Stroke. Journal of Neurology, Neurosurgery, and Psychiatry, 77, 1279-1281. https://doi.org/10.1136/jnnp.2006.100800

[20] Haeusler, K.G., et al. (2008) Cellular Immunodepression Preceding Infectious Complications after Acute Ischemic Stroke in Humans. Cerebrovascular Diseases, 25, 50-58. https://doi.org/10.1159/000111499

[21] Vogelgesang, A., et al. (2008) Analysis of Lymphocyte Subsets in Patients with 
Stroke and Their Influence on Infection after Stroke. Stroke, 39, 237-241. https://doi.org/10.1161/STROKEAHA.107.493635

[22] Tanzi, P., et al. (2011) Post-Stroke Infection: A Role for IL-1ra? Neurocrit Care, 14, 244-252. https://doi.org/10.1007/s12028-010-9490-7

[23] Prass, K., et al. (2003) Stroke-Induced Immunodeficiency Promotes Spontaneous Bacterial Infections and Is Mediated by Sympathetic Activation Reversal by Poststroke T Helper Cell Type 1-like Immunostimulation. The Journal of Experimental Medicine, 198, 725-736. https://doi.org/10.1084/jem.20021098

[24] Kalal, B.S. and Nagaraj, S. (2016) Urinary Tract Infections: A Retrospective, Descriptive Study of Causative Organisms and Antimicrobial Pattern of Samples Received for Culture, from a Tertiary Care Setting. Germs, 6, 132-138. https://doi.org/10.11599/germs.2016.1100

[25] Prakash, D. and Saxena, R.S. (2013) Distribution and Antimicrobial Susceptibility Pattern of Bacterial Pathogens Causing Urinary Tract Infection in Urban Community of Meerut City, India. ISRN Microbiology, 2013, Article ID: 749629.

[26] Dash, M., et al. (2013) Antimicrobial Resistance in Pathogens Causing Urinary Tract Infections in a Rural Community of Odisha, India. Journal of Family and Community Medicine, 20, 20-26. https://doi.org/10.4103/2230-8229.108180

[27] Fidel, P.L. (2002) Immunity to Candida. Oral Diseases, 8, 69-75. https://doi.org/10.1034/j.1601-0825.2002.00015.x

[28] Kunin, C.M. (2006) Urinary-Catheter-Associated Infections in the Elderly. International Journal of Antimicrobial Agents, 28, S78-S81. https://doi.org/10.1016/j.ijantimicag.2006.05.013

[29] Huang, W.C., et al. (2004) Catheter-Associated Urinary Tract Infections in Intensive Care Units Can Be Reduced by Prompting Physicians to Remove Unnecessary Catheters. Infection Control \& Hospital Epidemiology, 25, 974-978. https://doi.org/10.1086/502329

[30] Zorrilla-Vaca, A., et al. (2018) Acute Kidney Injury Following Acute Ischemic Stroke and Intracerebral Hemorrhage: A Meta-Analysis of Prevalence Rate and Mortality Risk. Cerebrovascular Diseases, 45, 1-9. https://doi.org/10.1159/000479338

[31] Donkor, E.S., et al. (2016) Post-Stroke Bacteriuria among Stroke Patients Attending a Physiotherapy Clinic in Ghana: A Cross-Sectional Study. Therapeutics and Clinical Risk Management, 12, 457-462. https://doi.org/10.2147/TCRM.S90474

[32] Matz, K., et al. (2006) Disorders of Glucose Metabolism in Acute Stroke Patients: An Underrecognized Problem. Diabetes Care, 29, 792-797. https://doi.org/10.2337/diacare.29.04.06.dc05-1818 